\title{
Article \\ Existence and uniqueness results for Navier problems with degenerated operators
}

\author{
Albo Carlos Cavalheiro',* \\ 1 State University of Londrina, Department of Mathematics, Londrina - PR, Brazil. \\ * Correspondence: accava@gmail.com
}

Received: 25 January 2019; Accepted: 9 February 2019; Published: 23 February 2019.

Abstract: In this article, we prove the existence and uniqueness of solutions for the Navier problem $\Delta\left[\omega_{1}(x)|\Delta u|^{p-2} \Delta u+v_{1}(x)|\Delta u|^{q-2} \Delta u\right]-\operatorname{div}\left[\omega_{2}(x)|\nabla u|^{p-2} \nabla u+v_{2}(x)|\nabla u|^{s-2} \nabla u\right]=f(x)-\operatorname{div}(G(x))$, in $\Omega$, with $u(x)=\Delta u=0$, in $\partial \Omega$, where $\Omega$ is a bounded open set of $\mathbb{R}^{N}$ for $N \geq 2, \frac{f}{\omega_{2}} \in L^{p^{\prime}}\left(\Omega, \omega_{2}\right)$ and $\frac{G}{v_{2}} \in\left[L^{s^{\prime}}\left(\Omega, v_{2}\right)\right]^{N}$.

Keywords: Degenerate nonlinear elliptic equations, weighted Sobolev space.

MSC: 35J60, 35J70.

\section{Introduction}

$\mathbf{T}$

he main purpose of this paper (see Theorem 7) is to establish the existence and uniqueness of solutions for the Navier problem

$$
(P)\left\{\begin{array}{l}
L u(x)=f(x)-\operatorname{div}(G(x)), \text { in } \Omega, \\
u(x)=\Delta u(x)=0, \text { in } \partial \Omega,
\end{array}\right.
$$

where

$$
\left.L u(x)=\Delta\left[\omega_{1}(x)|\Delta u|^{p-2} \Delta u+v_{1}(x)|\Delta u|^{q-2} \Delta u\right]-\operatorname{div}\left[\omega_{2}(x)|\nabla u|^{p-2} \nabla u+v_{2}(x)|\nabla u|^{s-2} \nabla u\right)\right]
$$

$\Omega \subset \mathbb{R}^{N}$ is a bounded open set, $\frac{f}{\omega_{2}} \in L^{p^{\prime}}\left(\Omega, \omega_{2}\right), \frac{G}{v_{2}} \in\left[L^{s^{\prime}}\left(\Omega, v_{2}\right)\right]^{N}, \omega_{1}, \omega_{2}, v_{1}$ and $v_{2}$ are four weight functions (i.e., $\omega_{i}$ and $v_{i}, i=1,2$ are locally integrable functions on $\mathbb{R}^{N}$ such that $0<\omega_{i}(x), v_{i}(x)<\infty$ a.e. $\left.x \in \mathbb{R}^{N}\right), \Delta$ is the Laplacian operator, $1<q, s<p<\infty, 1 / p+1 / p^{\prime}=1$ and $1 / s+1 / s^{\prime}=1$.

For degenerate partial differential equations, i.e., equations with various types of singularities in the coefficients, it is natural to look for solutions in weighted Sobolev spaces (see [1-8]). The type of a weight depends on the equation type.

A class of weights, which is particularly well understood, is the class of $A_{p}$ weights that was introduced by B.Muckenhoupt in the early 1970's (see [7]). These classes have found many useful applications in harmonic analysis (see [9] and [10]). Another reason for studying $A_{p}$-weights is the fact that powers of the distance to submanifolds of $\mathbb{R}^{N}$ often belong to $A_{p}$ (see [8] and [11]). There are, in fact, many interesting examples of weights (see [6] for p-admissible weights).

In the non-degenerate case (i.e. with $\omega(x) \equiv 1$ ), for all $f \in L^{p}(\Omega)$ the Poisson equation associated with the Dirichlet problem

$$
\left\{\begin{array}{l}
-\Delta u=f(x), \text { in } \Omega \\
u(x)=0, \text { in } \partial \Omega
\end{array}\right.
$$

is uniquely solvable in $W^{2, p}(\Omega) \cap W_{0}^{1, p}(\Omega)$ (see [12]), and the nonlinear Dirichlet problem

$$
\left\{\begin{array}{l}
-\Delta_{p} u=f(x), \text { in } \Omega \\
u(x)=0, \text { in } \partial \Omega
\end{array}\right.
$$


is uniquely solvable in $W_{0}^{1, p}(\Omega)$ (see [13]), where $\Delta_{p} u=\operatorname{div}\left(|\nabla u|^{p-2} \nabla u\right)$ is the p-Laplacian operator. In the degenerate case, the degenerated $p$-Laplacian has been studied in [11].

The paper is organized as follow. In Section 2 we present the definitions and basic results. In Section 3 we prove our main result about existence and uniqueness of solutions for problem $(P)$.

\section{Definitions and basic results}

Let $\Omega$ be an open set in $\mathbb{R}^{n}$. By the symbol $\mathcal{W}(\Omega)$ we denote the set of all measurable, a.e. in $\Omega$ positive and finite functions $\omega=\omega(x), x \in \Omega$. Elements of $\mathcal{W}(\Omega)$ will be called weight functions. Every weight $\omega$ gives rise to a measure on the measurable subsets of $\mathbb{R}^{N}$ through integration. This measure will be denoted by $\mu_{\omega}$. Thus, $\mu_{\omega}(E)=\int_{E} \omega(x) d x$ for measurable sets $E \subset \mathbb{R}^{N}$.

Definition 1. Let $1 \leq p<\infty$. A weight $\omega$ is said to be an $A_{p}$-weight, if there is a positive constant $C=C(p, \omega)$ such that, for every ball $B \subset \mathbb{R}^{N}$

$$
\begin{aligned}
& \left(\frac{1}{|B|} \int_{B} \omega(x) d x\right)\left(\frac{1}{|B|} \int_{B} \omega^{1 /(1-p)}(x) d x\right)^{p-1} \leq C, \text { if } p>1, \\
& \left(\frac{1}{|B|} \int_{B} \omega(x) d x\right)\left(\operatorname{ess} \sup _{x \in B} \frac{1}{\omega(x)}\right) \leq C, \text { if } p=1,
\end{aligned}
$$

where $|$.$| denotes the N$-dimensional Lebesgue measure in $\mathbb{R}^{N}$.

If $1<q \leq p$, then $A_{q} \subset A_{p}$ (see [5,6,8] for more information about $A_{p}$-weights). As an example of an $A_{p}$-weight, the function $\omega(x)=|x|^{\alpha}, x \in \mathbb{R}^{N}$, is in $A_{p}$ if and only if $-N<\alpha<N(p-1)$ (see [8], Chapter IX, Corollary 4.4). If $\varphi \in B M O\left(\mathbb{R}^{N}\right)$, then $\omega(x)=\mathrm{e}^{\alpha \varphi(x)} \in A_{2}$ for some $\alpha>0$ (see [9]).

Remark 1. If $\omega \in A_{p}, 1<p<\infty$, then

$$
\left(\frac{|E|}{|B|}\right)^{p} \leq C \frac{\mu_{\omega}(E)}{\mu_{\omega}(B)}
$$

for all measurable subsets $E$ of $B$ (see 15.5 strong doubling property in [6]). Therefore, $\mu_{\omega}(E)=0$ if and only if $|E|=0$; so there is no need to specify the measure when using the ubiquitous expression almost everywhere and almost every, both abbreviated a.e..

Definition 2. Let $\omega$ be a weight. We shall denote by $L^{p}(\Omega, \omega)(1 \leq p<\infty)$ the Banach space of all measurable functions $f$ defined in $\Omega$ for which

$$
\|f\|_{L^{p}(\Omega, \omega)}=\left(\int_{\Omega}|f(x)|^{p} \omega(x) d x\right)^{1 / p}<\infty .
$$

We denote $\left[L^{p}(\Omega, \omega)\right]^{N}=L^{p}(\Omega, \omega) \times \ldots \times L^{p}(\Omega, \omega)$.

Remark 2. If $\omega \in A_{p}, 1<p<\infty$, then since $\omega^{-1 /(p-1)}$ is locally integrable, we have $L^{p}(\Omega, \omega) \subset L_{\text {loc }}^{1}(\Omega)$ (see [8], Remark 1.2.4). It thus makes sense to talk about weak derivatives of functions in $L^{p}(\Omega, \omega)$.

Definition 3. Let $\Omega \subset \mathbb{R}^{N}$ be a bounded open set, $1<p<\infty, k$ be a nonnegative integer and $\omega \in A_{p}$. We shall denote by $W^{k, p}(\Omega, \omega)$, the weighted Sobolev spaces, the set of all functions $u \in L^{p}(\Omega, \omega)$ with weak derivatives $D^{\alpha} u \in L^{p}(\Omega, \omega), 1 \leq|\alpha| \leq k$. The norm in the space $W^{k, p}(\Omega, \omega)$ is defined by

$$
\|u\|_{W^{k, p}(\Omega, \omega)}=\left(\int_{\Omega}|u(x)|^{p} \omega(x) d x+\sum_{1 \leq|\alpha| \leq k} \int_{\Omega}\left|D^{\alpha} u(x)\right|^{p} \omega(x) d x\right)^{1 / p} .
$$

We also define the space $W_{0}^{k, p}(\Omega, \omega)$ as the closure of $C_{0}^{\infty}(\Omega)$ with respect to the norm (1). We have that the spaces $W^{k, p}(\Omega, \omega)$ and $W_{0}^{k, p}(\Omega, \omega)$ are Banach spaces (see Proposition 2.1.2 in [8]). 
The dual space of $W_{0}^{1, p}(\Omega, \omega)$ is the space $\left[W_{0}^{1, p}(\Omega, \omega)\right]^{*}=W^{-1, p^{\prime}}(\Omega, \omega)$,

$$
W^{-1, p^{\prime}}(\Omega, \omega)=\left\{T=f-\operatorname{div}(G): G=\left(g_{1}, \ldots, g_{N}\right), \frac{f}{\omega}, \frac{g_{j}}{\omega} \in L^{p^{\prime}}(\Omega, \omega)\right\} .
$$

It is evident that a weight function $\omega$ which satisfies $0<C_{1} \leq \omega(x) \leq C_{2}$, for a.e. $x \in \Omega$, gives nothing new (the space $\mathrm{W}^{k, p}(\Omega, \omega)$ is then identical with the classical Sobolev space $\mathrm{W}^{k, p}(\Omega)$ ). Consequently, we shall be interested in all above such weight functions $\omega$ which either vanish somewhere in $\Omega \cup \partial \Omega$ or increase to infinity (or both).

We need the following basics results.

Theorem 4. (The weighted Sobolev inequality) Let $\Omega \subset \mathbb{R}^{N}$ be a bounded open set and let $\omega$ be an $A_{p}$-weight, $1<p<$ $\infty$. Then there exists positive constants $C_{\Omega}$ and $\delta$ such that for all $u \in W_{0}^{1, p}(\Omega, \omega)$ and $1 \leq \eta \leq N /(N-1)+\delta$

$$
\|u\|_{L^{\eta p}(\Omega, \omega)} \leq C_{\Omega}\|\mid \nabla u\|_{L^{p}(\Omega, \omega)} .
$$

Proof. Its suffices to prove the inequality for functions $u \in C_{0}^{\infty}(\Omega)$ (see Theorem 1.3 in [4]). To extend the estimates (2) to arbitrary $u \in W_{0}^{1, p}(\Omega, \omega)$, we let $\left\{u_{m}\right\}$ be a sequence of $C_{0}^{\infty}(\Omega)$ functions tending to $u$ in $W_{0}^{1, p}(\Omega, \omega)$. Applying the estimates (2) to differences $u_{m_{1}}-u_{m_{2}}$, we see that $\left\{u_{m}\right\}$ will be a Cauchy sequence in $L^{p}(\Omega, \omega)$. Consequently the limit function $u$ will lie in the desired spaces and satisfy (2).

Lemma 5. (a) Let $1<p<\infty$, then exists a constant $C_{p}>0$ such that for all $\xi, \eta \in \mathbb{R}^{N}$,

$$
\left.|| \xi\right|^{p-2} \xi-|\eta|^{p-2} \eta\left|\leq C_{p}\right| \xi-\eta \mid(|\xi|+|\eta|)^{p-2} .
$$

(b) Let $1<p<\infty$. There exist two positive constants $\alpha_{p}$ and $\beta_{p}$ such that for every $\xi, \eta \in \mathbb{R}^{N}(N \geq 1)$

$$
\alpha_{p}(|\xi|+|\eta|)^{p-2}|\xi-\eta|^{2} \leq\left\langle|\xi|^{p-2} \xi-|\eta|^{p-2} \eta, \xi-\eta\right\rangle \leq \beta_{p}(|\xi|+|\eta|)^{p-2}|\xi-\eta|,
$$

where $\langle.,$.$\rangle denotes here the Euclidian scalar product in \mathbb{R}^{N}$.

Proof. See Proposition 17.2 and Proposition 17.3 in [13].

\section{Weak Solutions}

Let $\omega_{1}, \omega_{2} \in A_{p}$ and $v_{1}, v_{2} \in \mathcal{W}(\Omega), 1<q, s<p<\infty$. We denote by $X$ the space $X=W^{2, p}\left(\Omega, \omega_{1}\right) \cap W_{0}^{1, p}\left(\Omega, \omega_{2}\right)$ with the norm

$$
\|u\|_{X}=\left(\int_{\Omega}|\nabla u|^{p} \omega_{2} d x+\int_{\Omega}|\Delta u|^{p} \omega_{1} d x\right)^{1 / p}
$$

In this section we prove the existence and uniqueness of weak solutions $u \in X$ to the Navier problem

$$
(P)\left\{\begin{array}{l}
L u(x)=f(x)-\operatorname{div}(G(x)), \text { in } \Omega, \\
u(x)=\Delta u=0, \text { in } \partial \Omega
\end{array}\right.
$$

where $\Omega$ is a bounded open set of $\mathbb{R}^{N}(N \geq 2), \frac{f}{\omega_{2}} \in L^{p^{\prime}}\left(\Omega, \omega_{2}\right)$ and $\frac{G}{v_{2}} \in\left[L^{s^{\prime}}\left(\Omega, v_{2}\right)\right]^{N}, G=\left(g_{1}, \ldots, g_{N}\right)$.

Definition 6. We say that $u \in X$ is a weak solution for problem $(P)$ if

$$
\begin{aligned}
& \int_{\Omega}|\Delta u|^{p-2} \Delta u \Delta \varphi \omega_{1} d x+\int_{\Omega}|\Delta u|^{q-2} \Delta u \Delta \varphi v_{1} d x+\int_{\Omega}|\nabla u|^{p-2}\langle\nabla u, \nabla \varphi\rangle \omega_{2} d x \\
& +\int_{\Omega}|\nabla u|^{s-2}\langle\nabla u, \nabla \varphi\rangle v_{2} d x=\int_{\Omega} f \varphi d x+\int_{\Omega}\langle G, \nabla \varphi\rangle d x,
\end{aligned}
$$


for all $\varphi \in X$, with $f / \omega_{2} \in L^{p^{\prime}}\left(\Omega, \omega_{2}\right)$ and $G / v_{2} \in\left[L^{s^{\prime}}\left(\Omega, v_{2}\right)\right]^{N}$, where $\langle.,$.$\rangle denotes here the Euclidean scalar$ product in $\mathbb{R}^{N}$.

Remark 3. (a) Since $1<q, s<p<\infty$ and if $\frac{\nu_{1}}{\omega_{1}} \in L^{p /(p-q)}\left(\Omega, \omega_{1}\right)$ and $\frac{\nu_{2}}{\omega_{2}} \in L^{p /(p-s)}\left(\Omega, \omega_{2}\right)$, there exist two constants $M_{1}, M_{2}>0$ such that

$$
\|u\|_{L^{q}\left(\Omega, v_{1}\right)} \leq M_{1}\|u\|_{L^{p}\left(\Omega, \omega_{1}\right)} \text { and }\|u\|_{L^{s}\left(\Omega, v_{2}\right)} \leq M_{2}\|u\|_{L^{p}\left(\Omega, \omega_{2}\right)}
$$

where $M_{1}=\left[\int_{\Omega}\left(\frac{v_{1}}{\omega_{1}}\right) \omega_{1} d x\right]^{(p-q) / p q}$ and $M_{2}=\left[\int_{\Omega}\left(\frac{v_{2}}{\omega_{2}}\right) \omega_{2} d x\right]^{(p-s) / p s}$. In fact, since $1<q, s<p<\infty$, we have $r=p / q>1$ and $r^{\prime}=p /(p-q)$,

$$
\begin{aligned}
\|u\|_{L^{q}\left(\Omega, v_{1}\right)}^{q} & =\int_{\Omega}|u|^{q} v_{1} d x=\int_{\Omega}|u|^{q} \frac{v_{1}}{\omega_{1}} \omega_{1} d x \\
& \leq\left(\int_{\Omega}|u|^{q r} \omega_{1} d x\right)^{1 / r}\left(\int_{\Omega}\left(\frac{v_{1}}{\omega_{1}}\right)^{r^{\prime}} \omega_{1} d x\right)^{1 / r^{\prime}} \\
& =\left(\int_{\Omega}|u|^{p} \omega_{1} d x\right)^{q / p}\left(\int_{\Omega}\left(\frac{v_{1}}{\omega_{1}}\right)^{p /(p-q)} \omega_{1} d x\right)^{(p-q) / p} .
\end{aligned}
$$

Hence, $\|u\|_{L^{q}\left(\Omega, v_{1}\right)} \leq M_{1}\|u\|_{L^{p}\left(\Omega, \omega_{1}\right)}$. Analogously, we obtain $\|u\|_{L^{s}\left(\Omega, v_{2}\right)} \leq M_{2}\|u\|_{L^{p}\left(\Omega, \omega_{2}\right)}$. (b) Using the estimate in (a) we have

$$
\begin{aligned}
\left.\left|\int_{\Omega}\right| \Delta u\right|^{q-2} \Delta u \Delta \varphi v_{1} d x \mid & \leq \int_{\Omega}|\Delta u|^{q-1}|\Delta \varphi| v_{1} d x \\
& \leq\left(\int_{\Omega}|\Delta u|^{(q-1) q^{\prime}} v_{1} d x\right)^{1 / q^{\prime}}\left(\int_{\Omega}|\Delta \varphi|^{q} v_{1} d x\right)^{1 / q} \\
& =\left(\int_{\Omega}|\Delta u|^{q} v_{1} d x\right)^{(q-1) / q}\left(\int_{\Omega}|\Delta \varphi|^{q} v_{1} d x\right)^{1 / q} \\
& =\|\Delta u\|_{L^{q}\left(\Omega, v_{1}\right)}^{q-1}\|\Delta\|_{L^{q}\left(\Omega, v_{1}\right)} \\
& \leq M_{1}^{q-1}\|\Delta u\|_{L^{p}\left(\Omega, \omega_{1}\right)}^{q-1} M_{1}\|\Delta \varphi\|_{L^{p}\left(\Omega, \omega_{1}\right)} \\
& \leq M_{1}^{q}\|u\|_{X}\|\varphi\|_{X^{\prime}}
\end{aligned}
$$

and, analogously, we also have

$$
\left.\left|\int_{\Omega}\right| \nabla u\right|^{s-2}\langle\nabla u, \nabla \varphi\rangle v_{2} d x \mid \leq M_{2}^{s}\|u\|_{X}\|\varphi\|_{X}
$$

Theorem 7. Let $\omega_{i} \in A_{p}, v_{i} \in \mathcal{W}(\Omega)(i=1,2), 1<q, s<p<\infty$. Suppose that (a) $\frac{v_{1}}{\omega_{1}} \in L^{p /(p-q)}\left(\Omega, \omega_{1}\right)$ and $\frac{v_{2}}{\omega_{2}} \in L^{p /(p-s)}\left(\Omega, \omega_{2}\right)$;

(b) $f / \omega_{2} \in L^{p^{\prime}}\left(\Omega, \omega_{2}\right)$ and $G / \nu_{2} \in\left[L^{s^{\prime}}\left(\Omega, v_{2}\right)\right]^{N}$.

Then the problem $(P)$ has a unique solution $u \in X$ and

$$
\|u\|_{X} \leq\left[C_{\Omega}\left\|\frac{f}{\omega_{2}}\right\|_{L^{p^{\prime}\left(\Omega, \omega_{2}\right)}}+M_{2}\left\|\frac{|G|}{v_{2}}\right\|_{L^{s^{\prime}}\left(\Omega, v_{2}\right)}\right]^{1 /(p-1)},
$$

where $C_{\Omega}$ is the constant in Theorem 4 and $M_{2}$ is the constant in Remark 3 (a).

Proof. (I) Existence. By Theorem 4 (with $\eta=1$ ), we have that

$$
\left|\int_{\Omega} f \varphi d x\right| \leq\left(\int_{\Omega}\left|\frac{f}{\omega_{2}}\right|^{p^{\prime}} \omega_{2} d x\right)^{1 / p^{\prime}}\left(\int_{\Omega}|\varphi|^{p} \omega_{2} d x\right)^{1 / p}
$$




$$
\leq C_{\Omega}\left\|\frac{f}{\omega_{2}}\right\|_{L^{p^{\prime}}\left(\Omega, \omega_{2}\right)}\||\nabla \varphi|\|_{L^{p}\left(\Omega, \omega_{2}\right)} \leq C_{\Omega}\left\|\frac{f}{\omega_{2}}\right\|_{L^{p^{\prime}}(\Omega, \omega)}\|\varphi\|_{X^{\prime}}
$$

and by Remark 3 (a)

$$
\begin{aligned}
\left|\int_{\Omega}\langle G, \nabla \varphi\rangle d x\right| d x & \leq \int_{\Omega}|\langle G, \nabla \varphi\rangle| d x \leq \int_{\Omega}|G||\nabla \varphi| d x=\int_{\Omega} \frac{|G|}{v_{2}}|\nabla \varphi| v_{2} d x \\
& \leq\left\|\frac{|G|}{v_{2}}\right\|_{L^{s^{\prime}}\left(\Omega, v_{2}\right)}\||\nabla \varphi|\|_{L^{s}\left(\Omega, v_{2}\right)} \leq M_{2}\left\|\frac{|G|}{v_{2}}\right\|_{L^{s^{\prime}}\left(\Omega, v_{2}\right)}\||\nabla \varphi|\|_{L^{p}\left(\Omega, \omega_{2}\right)} \\
& \leq M_{2}\left\|\frac{|G|}{v_{2}}\right\|_{L^{s^{\prime}}\left(\Omega, v_{2}\right)}\|\varphi\|_{X} .
\end{aligned}
$$

Define the functional $J: X \rightarrow \mathbb{R}$ by

$$
\begin{aligned}
J(\varphi)= & \frac{1}{p} \int_{\Omega}|\Delta \varphi|^{p} \omega_{1} d x+\frac{1}{q} \int_{\Omega}|\Delta \varphi|^{q} v_{1} d x \\
& +\frac{1}{p} \int_{\Omega}|\nabla \varphi|^{p} \omega_{2} d x+\frac{1}{s} \int_{\Omega}|\nabla \varphi|^{s} v_{2} d x-\int_{\Omega} f \varphi d x-\int_{\Omega}\langle G, \nabla \varphi\rangle d x .
\end{aligned}
$$

Using (4), (5), Remark 3(a) and Young's inequality $\left(a b \leq \frac{a^{p}}{p}+\frac{b^{p^{\prime}}}{p^{\prime}}\right)$, we have that

$$
\begin{aligned}
J(\varphi) \geq & \frac{1}{p} \int_{\Omega}|\Delta \varphi|^{p} \omega_{1} d x+\frac{1}{q} \int_{\Omega}|\Delta \varphi|^{q} v_{1} d x+\frac{1}{p} \int_{\Omega}|\nabla \varphi|^{p} \omega_{2} d x+\frac{1}{s} \int_{\Omega}|\nabla \varphi|^{s} v_{2} d x \\
& -\left\|\frac{f}{\omega_{2}}\right\|_{L^{p^{\prime}}\left(\Omega, \omega_{2}\right)}\|\varphi\|_{L^{p}\left(\Omega, \omega_{2}\right)}-\left\|\frac{|G|}{v_{2}}\right\|_{L^{s^{\prime}}\left(\Omega, v_{2}\right)}\||\nabla \varphi|\|_{L^{s}\left(\Omega, v_{2}\right)}\left\|\frac{1}{\geq} \int_{\Omega}|\nabla \varphi|^{p} \omega_{2} d x+\frac{1}{s} \int_{\Omega}|\nabla \varphi|^{s} v_{2} d x-C_{\Omega}\right\| \frac{f}{\omega_{2}}\left\|_{L^{p^{\prime}}\left(\Omega, \omega_{2}\right)}\right\||\nabla \varphi| \|_{L^{p}\left(\Omega, \omega_{2}\right)} \\
& -\left\|\frac{|G|}{v_{2}}\right\|_{L^{s^{\prime}}\left(\Omega, v_{2}\right)}\||\nabla \varphi|\|_{L^{s}\left(\Omega, v_{2}\right)} \\
\geq & \frac{1}{p} \int_{\Omega}|\nabla \varphi|^{p} \omega_{2} d x+\frac{1}{s} \int_{\Omega}|\nabla \varphi|^{s} v_{2} d x-\frac{C_{\Omega}^{p^{\prime}}}{p^{\prime}}\left\|\frac{f}{\omega_{2}}\right\|_{L^{p^{\prime}}\left(\Omega, \omega_{2}\right)}^{p^{\prime}} \\
& -\frac{1}{p}\||\nabla \varphi|\|_{L^{p}\left(\Omega, \omega_{2}\right)}^{p}-\frac{1}{s^{\prime}}\left\|\frac{|G|}{v_{2}}\right\|_{L^{s^{\prime}}\left(\Omega, v_{2}\right)}^{s^{\prime}}-\frac{1}{s}\||\nabla \varphi|\|_{L^{s}\left(\Omega, v_{2}\right)}^{s} \\
= & -\frac{C_{\Omega}^{p^{\prime}}}{p^{\prime}}\left\|\frac{f}{\omega_{2}}\right\|_{L^{p^{\prime}}\left(\Omega, \omega_{2}\right)}^{p^{\prime}}-\frac{1}{s^{\prime}}\left\|\frac{|G|}{v_{2}}\right\|_{L^{s^{\prime}}\left(\Omega, v_{2}\right)}^{s^{\prime}}
\end{aligned}
$$

that is, $J$ is bounded from below. Let $\left\{u_{n}\right\}$ be a minimizing sequence, that is, a sequence such that

$$
J\left(u_{n}\right) \rightarrow \inf _{\varphi \in X} J(\varphi) .
$$

Then for $n$ large enough, we obtain

$$
\begin{aligned}
0 \geq J\left(u_{n}\right) & =\frac{1}{p} \int_{\Omega}\left|\Delta u_{n}\right|^{p} \omega_{1} d x+\frac{1}{q} \int_{\Omega}\left|\Delta u_{n}\right|^{q} v_{1} d x+\frac{1}{p} \int_{\Omega}\left|\nabla u_{n}\right|^{p} \omega_{2} d x+\frac{1}{s} \int_{\Omega}\left|\nabla u_{n}\right|^{s} v_{2} d x \\
& -\int_{\Omega} f u_{n} d x-\int_{\Omega}\left\langle G, \nabla u_{n}\right\rangle d x
\end{aligned}
$$

and we have

$$
\frac{1}{p} \int_{\Omega}\left|\Delta u_{n}\right|^{p} \omega_{1} d x+\frac{1}{p} \int_{\Omega}\left|\nabla u_{n}\right|^{p} \omega_{2} d x
$$




$$
\begin{aligned}
& \leq \frac{1}{p} \int_{\Omega}\left|\Delta u_{n}\right|^{p} \omega_{1} d x+\frac{1}{q} \int_{\Omega}\left|\Delta u_{n}\right|^{q} v_{1} d x+\frac{1}{p} \int_{\Omega}\left|\nabla u_{n}\right|^{p} \omega_{2} d x+\frac{1}{s} \int_{\Omega}\left|\nabla u_{n}\right|^{s} v_{2} d x \\
& \leq \int_{\Omega} f u_{n} d x+\int_{\Omega}\left\langle G, u_{n}\right\rangle d x .
\end{aligned}
$$

Hence, by Theorem 4 (with $\eta=1$ ), Remark 3(a) and (6), we obtain

$$
\begin{aligned}
\left\|u_{n}\right\|_{X}^{p}= & \int_{\Omega}\left|\Delta u_{n}\right|^{p} \omega_{1} d x+\int_{\Omega}\left|\nabla u_{n}\right|^{p} \omega_{2} d x \\
& \leq p\left(\int_{\Omega} f u_{n} d x+\int_{\Omega}\left\langle G, \nabla u_{n}\right\rangle d x\right) \\
& \leq p\left(\left\|\frac{f}{\omega_{2}}\right\|_{L^{p^{\prime}}\left(\Omega, \omega_{2}\right)}\left\|u_{n}\right\|_{L^{p}\left(\Omega, \omega_{2}\right)}+\left\|\frac{|G|}{v_{2}}\right\|_{L^{s^{\prime}}\left(\Omega, v_{2}\right)}\left\|\left|\nabla u_{n}\right|\right\| \|_{L^{s}\left(\Omega, v_{2}\right)}\right) \\
& \leq p\left(C_{\Omega}\left\|\frac{f}{\omega_{2}}\right\|_{L^{p^{\prime}\left(\Omega, \omega_{2}\right)}}\left\|\left|\nabla u_{n}\right|\right\|_{L^{p}\left(\Omega, \omega_{2}\right)}+M_{2}\left\|\frac{|G|}{v_{2}}\right\|_{L^{s^{\prime}}\left(\Omega, v_{2}\right)}\left\|\left|\nabla u_{n}\right|\right\|_{L^{p}\left(\Omega, \omega_{2}\right)}\right) \\
& \leq p\left(C_{\Omega}\left\|\frac{f}{\omega_{2}}\right\|_{L^{p^{\prime}\left(\Omega, \omega_{2}\right)}}+M_{2}\left\|\frac{|G|}{v_{2}}\right\|_{L^{q^{\prime}}\left(\Omega, v_{2}\right)}\right)\left\|u_{n}\right\|_{X} .
\end{aligned}
$$

Hence,

$$
\left\|u_{n}\right\|_{X} \leq\left[p\left(C_{\Omega}\left\|\frac{f}{\omega_{2}}\right\|_{L^{p^{\prime}\left(\Omega, \omega_{2}\right)}}+M_{2}\left\|\frac{|G|}{v_{2}}\right\|_{L^{s^{\prime}}\left(\Omega, v_{2}\right)}\right)\right]^{1 /(p-1)} .
$$

Therefore $\left\{u_{n}\right\}$ is bounded in $X$. Since $X$ is reflexive, there exists a subsequence, still denoted by $\left\{u_{n}\right\}$, and a function $u \in X$ such that $u_{n} \rightarrow u$ in $X$. Since,

$$
X \ni \varphi \mapsto \int_{\Omega} f \varphi d x+\int_{\Omega}\langle G, \nabla \varphi\rangle d x,
$$

and

$$
X \ni \varphi \mapsto\|\Delta \varphi\|_{L^{p}\left(\Omega, \omega_{1}\right)}^{p}+\|\Delta \varphi\|_{L^{q}\left(\Omega, v_{1}\right)}^{q}+\||\nabla \varphi|\|_{L^{p}\left(\Omega, \omega_{2}\right)}^{p}+\||\nabla \varphi|\|_{L^{s}\left(\Omega, v_{2}\right)}^{s},
$$

are continuous then $J$ is continuous. Moreover since $1<q, s<p<\infty$ we have that $J$ is convex and thus lower semi-continuous for the weak convergence. It follows that

$$
J(u) \leq \liminf _{n} J\left(u_{n}\right)=\inf _{\varphi \in X} J(\varphi),
$$

and thus $u$ is a minimizer of $J$ on $X$ (see Theorem 25.C and Corollary 25.15 in [14]). For any $\varphi \in X$ the function

$$
\begin{aligned}
\lambda \mapsto \quad & \frac{1}{p} \int_{\Omega}|\Delta(u+\lambda \varphi)|^{p} \omega_{1} d x+\frac{1}{q} \int_{\Omega}|\Delta(u+\lambda \varphi)|^{q} v_{1} d x+\frac{1}{p} \int_{\Omega}|\nabla(u+\lambda \varphi)|^{p} \omega_{2} d x \\
& +\frac{1}{s} \int_{\Omega}|\nabla(u+\lambda \varphi)|^{s} v_{2} d x-\int_{\Omega}(u+\lambda \varphi) f d x-\int_{\Omega}\langle G, \nabla(u+\lambda \varphi)\rangle d x
\end{aligned}
$$

has a minimum at $\lambda=0$. Hence,

$$
\left.\frac{d}{d \lambda}(J(u+\lambda \varphi))\right|_{\lambda=0}=0, \forall \varphi \in X
$$

We have

$$
\frac{d}{d \lambda}\left(|\nabla(u+\lambda \varphi)|^{p} \omega_{2}\right)=p\left\{|\nabla(u+\lambda \varphi)|^{p-2}\left(\langle\nabla u, \nabla \varphi\rangle+\lambda|\nabla \varphi|^{2}\right)\right\} \omega_{2},
$$

and

$$
\frac{d}{d \lambda}\left(|\Delta(u+\lambda \varphi)|^{p} \omega_{1}\right)=p|\Delta u+\lambda \Delta \varphi|^{p-2}(\Delta u+\lambda \Delta \varphi) \Delta \varphi \omega_{1}
$$

and we obtain

$$
0=\left.\frac{d}{d \lambda}(J(u+\lambda \varphi))\right|_{\lambda=0}=\left[\frac { 1 } { p } \left(p \int_{\Omega}|\nabla(u+\lambda \varphi)|^{p-2}\left(\langle\nabla u, \nabla \varphi\rangle+\lambda|\nabla \varphi|^{2}\right) \omega_{2} d x\right.\right.
$$




$$
\begin{aligned}
& \left.+p \int_{\Omega}|\Delta u+\lambda \Delta \varphi|^{p-2}(\Delta u+\lambda \Delta \varphi) \Delta \varphi \omega_{1} d x\right)+\frac{1}{s}\left(s \int_{\Omega}|\nabla(u+\lambda \varphi)|^{s-2}\left(\langle\nabla u, \nabla \varphi\rangle+\lambda|\nabla \varphi|^{2}\right) v_{2} d x\right) \\
& \left.+\frac{1}{q}\left(q \int_{\Omega}|\Delta u+\lambda \Delta \varphi|^{q-2}(\Delta u+\lambda \Delta \varphi) \Delta \varphi v_{1} d x\right)-\int_{\Omega} \varphi f d x-\int_{\Omega}\langle G, \nabla \varphi\rangle d x\right]\left.\right|_{\lambda=0} \\
& =\int_{\Omega}|\Delta u|^{p-2} \Delta u \Delta \varphi \omega_{1} d x+\int_{\Omega}|\nabla u|^{p-2}\langle\nabla u, \nabla \varphi\rangle \omega_{2} d x+\int_{\Omega}|\Delta u|^{q-2} \Delta u \Delta \varphi v_{1} d x \\
& +\int_{\Omega}|\nabla u|^{s-2}\langle\nabla u, \nabla \varphi\rangle v_{2} d x-\int_{\Omega} f \varphi d x-\int_{\Omega}\langle G, \nabla \varphi\rangle d x .
\end{aligned}
$$

Therefore

$$
\begin{aligned}
& \int_{\Omega}|\Delta u|^{p-2} \Delta u \Delta \varphi \omega_{1} d x+\int_{\Omega}|\nabla u|^{p-2}\langle\nabla u \nabla \varphi\rangle \omega_{2} d x+\int_{\Omega}|\Delta u|^{q-2} \Delta u \Delta \varphi v_{1} d x+\int_{\Omega}|\nabla u|^{s-2}\langle\nabla u, \nabla \varphi\rangle v_{2} d x \\
& =\int_{\Omega} f \varphi d x+\int_{\Omega}\langle G, \nabla \varphi\rangle d x,
\end{aligned}
$$

for all $\varphi \in X$, that is, $u \in X$ is a solution of problem $(P)$.

(II) Uniqueness. If $u_{1}, u_{2} \in X$ are two weak solutions of problem $(P)$, we have

$$
\begin{aligned}
& \int_{\Omega}\left|\Delta u_{1}\right|^{p-2} \Delta u_{1} \Delta \varphi \omega_{1} d x+\int_{\Omega}\left|\Delta u_{1}\right|^{q-2} \Delta u_{1} \Delta \varphi v_{1} d x+\int_{\Omega}\left|\nabla u_{1}\right|^{p-2}\left\langle\nabla u_{1}, \nabla \varphi\right\rangle \omega_{2} d x \\
& +\int_{\Omega}\left|\nabla u_{1}\right|^{s-2}\left\langle\nabla u_{1}, \nabla \varphi\right\rangle v_{2} d x=\int_{\Omega} f \varphi d x+\int_{\Omega}\langle G, \nabla \varphi\rangle d x,
\end{aligned}
$$

and

$$
\begin{aligned}
& \int_{\Omega}\left|\Delta u_{2}\right|^{p-2} \Delta u_{2} \Delta \varphi \omega_{1} d x+\int_{\Omega}\left|\Delta u_{2}\right|^{q-2} \Delta u_{2} \Delta \varphi v_{1} d x+\int_{\Omega}\left|\nabla u_{2}\right|^{p-2}\left\langle\nabla u_{2}, \nabla \varphi\right\rangle \omega_{2} d x \\
& +\int_{\Omega}\left|\nabla u_{2}\right|^{s-2}\left\langle\nabla u_{2}, \nabla \varphi\right\rangle v_{2} d x=\int_{\Omega} f \varphi d x+\int_{\Omega}\langle G, \nabla \varphi\rangle d x,
\end{aligned}
$$

for all $\varphi \in X$. Hence

$$
\begin{aligned}
& \int_{\Omega}\left(\left|\Delta u_{1}\right|^{p-2} \Delta u_{1}-\left|\Delta u_{2}\right|^{p-2} \Delta u_{2}\right) \Delta \varphi \omega_{1} d x+\int_{\Omega}\left(\left|\Delta u_{1}\right|^{q-2} \Delta u_{1}-\left|\Delta u_{2}\right|^{q-2} \Delta u_{2}\right) \Delta \varphi v_{1} d x \\
& +\int_{\Omega}\left(\left|\nabla u_{1}\right|^{p-2}\left\langle\nabla u_{1}, \nabla \varphi\right\rangle-\left|\nabla u_{2}\right|^{p-2}\left\langle\nabla u_{2}, \nabla \varphi\right\rangle\right) \omega_{2} d x+\int_{\Omega}\left(\left|\nabla u_{1}\right|^{s-2}\left\langle\nabla u_{1}, \nabla \varphi\right\rangle\right. \\
& \left.-\left|\nabla u_{2}\right|^{s-2}\left\langle\nabla u_{2}, \nabla \varphi\right\rangle\right) v_{2} d x=0 .
\end{aligned}
$$

Taking $\varphi=u_{1}-u_{2}$, and using Lemma 5 (b) there exist positive constants $\alpha_{p}, \tilde{\alpha}_{p}, \alpha_{q}, \alpha_{s}$ such that

$$
\begin{aligned}
0= & \int_{\Omega}\left(\left|\Delta u_{1}\right|^{p-2} \Delta u_{1}-\left|\Delta u_{2}\right|^{p-2} \Delta u_{2}\right)\left(\Delta u_{1}-\Delta u_{2}\right) \omega_{1} d x \\
& +\int_{\Omega}\left(\left|\Delta u_{1}\right|^{q-2} \Delta u_{1}-\left|\Delta u_{2}\right|^{q-2} \Delta u_{2}\right)\left(\Delta u_{1}-\Delta u_{2}\right) v_{1} d x \\
& +\int_{\Omega}\left(\left|\nabla u_{1}\right|^{p-2}\left\langle\nabla u_{1}, \nabla u_{1}-\nabla u_{2}\right\rangle-\left|\nabla u_{2}\right|^{p-2}\left\langle\nabla u_{2}, \nabla u_{1}-\nabla u_{2}\right\rangle\right) \omega_{2} d x \\
& +\int_{\Omega}\left(\left|\nabla u_{1}\right|^{s-2}\left\langle\nabla u_{1}, \nabla u_{1}-\nabla u_{2}\right\rangle-\left|\nabla u_{2}\right|^{s-2}\left\langle\nabla u_{2}, \nabla u_{1}-\nabla u_{2}\right\rangle\right) v_{2} d x \\
= & \int_{\Omega}\left(\left|\Delta u_{1}\right|^{p-2} \Delta u_{1}-\left|\Delta u_{2}\right|^{p-2} \Delta u_{2}\right)\left(\Delta u_{1}-\Delta u_{2}\right) \omega_{1} d x \\
& +\int_{\Omega}\left(\left|\Delta u_{1}\right|^{q-2} \Delta u_{1}-\left|\Delta u_{2}\right|^{q-2} \Delta u_{2}\right)\left(\Delta u_{1}-\Delta u_{2}\right) v_{1} d x \\
& +\int_{\Omega}\left\langle\left|\nabla u_{1}\right|^{p-2} \nabla u_{1}-\left|\nabla u_{2}\right|^{p-2} \nabla u_{2}, \nabla u_{1}-\nabla u_{2}\right\rangle \omega_{2} d x
\end{aligned}
$$




$$
\begin{aligned}
& +\int_{\Omega}\left\langle\left|\nabla u_{1}\right|^{s-2} \nabla u_{1}-\left|\nabla u_{2}\right|^{s-2} \nabla u_{2}, \nabla u_{1}-\nabla u_{2}\right\rangle v_{2} d x \\
\geq & \alpha_{p} \int_{\Omega}\left(\left|\Delta u_{1}\right|+\left|\Delta u_{2}\right|\right)^{p-2}\left|\Delta u_{1}-\Delta u_{2}\right|^{2} \omega_{1} d x+\tilde{\alpha}_{p} \int_{\Omega}\left(\left|\nabla u_{1}\right|+\left|\nabla u_{2}\right|\right)^{p-2}\left|\nabla u_{1}-\nabla u_{2}\right|^{2} \omega_{2} d x \\
& +\alpha_{q} \int_{\Omega}\left(\left|\Delta u_{1}\right|+\left|\Delta u_{2}\right|\right)^{q-2}\left|\Delta u_{1}-\Delta u_{2}\right|^{2} v_{1} d x+\alpha_{s} \int_{\Omega}\left(\left|\nabla u_{1}\right|+\left|\nabla u_{2}\right|\right)^{s-2}\left|\nabla u_{1}-\nabla u_{2}\right|^{2} v_{2} d x \\
\geq & \alpha_{p} \int_{\Omega}\left(\left|\Delta u_{1}\right|+\left|\Delta u_{2}\right|\right)^{p-2}\left|\Delta u_{1}-\Delta u_{2}\right|^{2} \omega_{1} d x+\tilde{\alpha}_{p} \int_{\Omega}\left(\left|\nabla u_{1}\right|+\left|\nabla u_{2}\right|\right)^{p-2}\left|\nabla u_{1}-\nabla u_{2}\right|^{2} \omega_{2} d x .
\end{aligned}
$$

Therefore $\Delta u_{1}=\Delta u_{2}$ and $\nabla u_{1}=\nabla u_{2}$ a.e. and since $u_{1}, u_{2} \in X$, then $u_{1}=u_{2}$ a.e. (by Remark 1 ).

(III) Estimate for $\|u\|_{X}$.

In particular, for $\varphi=u \in X$ in Definition 6 we have

$$
\int_{\Omega}|\Delta u|^{p} \omega_{1} d x+\int_{\Omega}|\Delta u|^{q} v_{1} d x+\int_{\Omega}|\nabla u|^{p} \omega_{2} d x+\int_{\Omega}|\nabla u|^{s} v_{2} d x=\int_{\Omega} f u d x+\int_{\Omega}\langle G, \nabla u\rangle d x .
$$

Then, by Theorem 4 and Remark 3(a), we obtain

$$
\begin{aligned}
\|u\|_{X}^{p} & =\int_{\Omega}|\Delta u|^{p} \omega_{1} d x+\int_{\Omega}|\nabla u|^{p} \omega_{2} d x \\
& \leq \int_{\Omega}|\Delta u|^{p} \omega_{1} d x+\int_{\Omega}|\Delta u|^{q} v_{1} d x+\int_{\Omega}|\nabla u|^{p} \omega_{2} d x+\int_{\Omega}|\nabla u|^{s} v_{2} d x \\
& =\int_{\Omega} f u d x+\int_{\Omega}\langle G, \nabla u\rangle d x \\
& \leq\left\|\frac{f}{\omega_{2}}\right\|_{L^{p^{\prime}}\left(\Omega, \omega_{2}\right)}\|u\|_{L^{p}\left(\Omega, \omega_{2}\right)}+\left\|\frac{|G|}{v_{2}}\right\|_{L^{s^{\prime}}\left(\Omega, v_{2}\right)}\||\nabla u|\|_{L^{s}\left(\Omega, v_{2}\right)} \\
& \leq C_{\Omega}\left\|\frac{f}{\omega_{2}}\right\|_{L^{p^{\prime}}\left(\Omega, \omega_{2}\right)}\||\nabla u|\|_{L^{p}\left(\Omega, \omega_{2}\right)}+M_{2}\left\|\frac{|G|}{v_{2}}\right\|_{L^{s^{\prime}}\left(\Omega, v_{2}\right)}\||\nabla u|\|_{L^{p}\left(\Omega, \omega_{2}\right)} \\
& \leq\left(C_{\Omega}\left\|\frac{f}{\omega_{2}}\right\|_{L^{p^{\prime}}\left(\Omega, \omega_{2}\right)}+M_{2}\left\|\frac{|G|}{v_{2}}\right\|_{L^{s^{\prime}}\left(\Omega, v_{2}\right)}\right)\|u\|_{X} .
\end{aligned}
$$

Therefore,

$$
\|u\|_{X} \leq\left(C_{\Omega}\left\|\frac{f}{\omega_{2}}\right\|_{L^{p^{\prime}\left(\Omega, \omega_{2}\right)}}+M_{2}\left\|\frac{|G|}{v_{2}}\right\|_{L^{s^{\prime}}\left(\Omega, v_{2}\right)}\right)^{1 /(p-1)} .
$$

Corollary 8. Under the assumptions of Theorem 7 with $2 \leq q, s<p<\infty$. If $u_{1}, u_{2} \in X$ are solutions of

$$
\left(P_{1}\right)\left\{\begin{array}{l}
L u_{1}(x)=f(x)-\operatorname{div}(G(x)), \text { in } \Omega, \\
u_{1}(x)=\Delta u_{1}(x)=0, \text { in } \partial \Omega,
\end{array}\right.
$$

and

$$
\left(P_{2}\right)\left\{\begin{array}{l}
L u_{2}(x)=\tilde{f}(x)-\operatorname{div}(\tilde{G}(x)), \text { in } \Omega, \\
u_{2}(x)=\Delta u_{2}(x)=0, \text { in } \partial \Omega,
\end{array}\right.
$$

then

$$
\left\|u_{1}-u_{2}\right\|_{X} \leq \frac{1}{\gamma^{1 /(p-1)}}\left(C_{\Omega}\left\|\frac{f-\tilde{f}}{\omega_{2}}\right\|_{L^{p^{\prime}}\left(\Omega, \omega_{2}\right)}+M_{2}\left\|\frac{|G-\tilde{G}|}{v_{2}}\right\|_{L^{s^{\prime}}\left(\Omega, v_{2}\right)}\right)^{1 /(p-1)},
$$

where $\gamma$ is a positive constant, $C_{\Omega}$ and $M_{2}$ are the same constants of Theorem 7 .

Proof. If $u_{1}$ and $u_{2}$ are solutions of $(P 1)$ and (P2) then for all $\varphi \in X$ we have

$$
\int_{\Omega}\left|\Delta u_{1}\right|^{p-2} \Delta u_{1} \Delta \varphi \omega_{1} d x+\int_{\Omega}\left|\Delta u_{1}\right|^{q-2} \Delta u_{1} \Delta \varphi v_{1} d x+\int_{\Omega}\left|\nabla u_{1}\right|^{p-2}\left\langle\nabla u_{1}, \nabla \varphi\right\rangle \omega_{2} d x
$$




$$
\begin{aligned}
& +\int_{\Omega}\left|\nabla u_{1}\right|^{s-2}\left\langle\nabla u_{1}, \nabla \varphi\right\rangle v_{2} d x-\left(\int_{\Omega}\left|\Delta u_{2}\right|^{p-2} \Delta u_{2} \Delta \varphi \omega_{1} d x+\int_{\Omega}\left|\Delta u_{2}\right|^{q-2} \Delta u_{2} \Delta \varphi v_{1} d x\right. \\
& \left.+\int_{\Omega}\left|\nabla u_{1}\right|^{p-2}\left\langle\nabla u_{2}, \nabla \varphi\right\rangle \omega_{2} d x+\int_{\Omega}\left|\nabla u_{2}\right|^{s-2}\left\langle\nabla u_{2}, \nabla \varphi\right\rangle v_{2} d x\right)=\int_{\Omega}(f-\tilde{f}) \varphi d x+\int_{\Omega}\langle G-\tilde{G}, \nabla \varphi\rangle d x .
\end{aligned}
$$

In particular, for $\varphi=u_{1}-u_{2}$, we obtain in (7).

(i) By Lemma 5 (b) and since $2 \leq q, s<p<\infty$, there exist two positive constants $\alpha_{p}$ and $\alpha_{q}$ such that

$$
\begin{aligned}
& \int_{\Omega}\left(\left|\Delta u_{1}\right|^{p-2} \Delta u_{1}-\left|\Delta_{2}\right|^{p-2} \Delta u_{2}\right) \Delta\left(u_{1}-u_{2}\right) \omega_{1} d x \geq \alpha_{p} \int_{\Omega}\left(\left|\Delta u_{1}\right|+\left|\Delta u_{2}\right|\right)^{p-2}\left|\Delta u_{1}-\Delta u_{2}\right|^{2} \omega_{1} d x \\
& \geq \alpha_{p} \int_{\Omega}\left|\Delta u_{1}-\Delta u_{2}\right|^{p-2}\left|\Delta u_{1}-\Delta u_{2}\right|^{2} \omega_{1} d x=\alpha_{p} \int_{\Omega}\left|\Delta\left(u_{1}-u_{2}\right)\right|^{p} \omega_{1} d x,
\end{aligned}
$$

and analogously

$$
\int_{\Omega}\left(\left|\Delta u_{1}\right|^{q-2} \Delta u_{1}-\left|\Delta u_{2}\right|^{q-2} \Delta u_{2}\right) \Delta\left(u_{1}-u_{2}\right) v_{1} d x \geq \alpha_{q} \int_{\Omega}\left|\Delta\left(u_{1}-u_{2}\right)\right|^{q} v_{1} d x \geq 0 .
$$

(ii) Since $2 \leq q, s<p<\infty$ and by Lemma 5(b), there exit two positive constants $\tilde{\alpha}_{p}$ and $\alpha_{s}$ such that

$$
\begin{aligned}
& \int_{\Omega}\left(\left|\nabla u_{1}\right|^{p-2}\left\langle\nabla u_{1}, \nabla\left(u_{1}-u_{2}\right)\right\rangle-\left|\nabla u_{2}\right|^{p-2}\left\langle\nabla u_{2}, \nabla\left(u_{1}-u_{2}\right)\right\rangle\right) \omega_{2} d x \\
& =\int_{\Omega}\left\langle\left|\nabla u_{1}\right|^{p-2} \nabla u_{1}-\left|\nabla u_{2}\right|^{p-2} \nabla u_{2}, \nabla\left(u_{1}-u_{2}\right)\right\rangle \omega_{2} d x \\
& \geq \tilde{\alpha}_{p} \int_{\Omega}\left(\left|\nabla u_{1}\right|+\left|\nabla u_{2}\right|\right)^{p-2}\left|\nabla u_{1}-\nabla u_{2}\right|^{2} \omega_{2} d x \\
& \geq \tilde{\alpha}_{p} \int_{\Omega}\left|\nabla u_{1}-\nabla u_{2}\right|^{p-2}\left|\nabla u_{1}-\nabla u_{2}\right|^{2} \omega_{2} d x=\tilde{\alpha}_{p} \int_{\Omega}\left|\nabla\left(u_{1}-u_{2}\right)\right|^{p} \omega_{2} d x,
\end{aligned}
$$

and analogously,

$$
\int_{\Omega}\left(\left|\nabla u_{1}\right|^{s-2}\left\langle\nabla u_{1}, \nabla\left(u_{1}-u_{2}\right)\right\rangle-\left|\nabla u_{2}\right|^{s-2}\left\langle\nabla u_{2}, \nabla\left(u_{1}-u_{2}\right)\right\rangle\right) v_{2} d x \geq \alpha_{s} \int_{\Omega}\left|\nabla\left(u_{1}-u_{2}\right)\right|^{s} v_{2} d x \geq 0 .
$$

(iii) By Remark 3 (a) we have

$$
\begin{aligned}
& \left|\int_{\Omega}(f-\tilde{f})\left(u_{1}-u_{2}\right) d x+\int_{\Omega}\left\langle G-\tilde{G}, \nabla\left(u_{1}-u_{2}\right)\right\rangle d x\right| \\
& \leq\left(C_{\Omega}\left\|\frac{f-\tilde{f}}{\omega_{2}}\right\|_{L^{p^{\prime}\left(\Omega, \omega_{2}\right)}}+M_{2}\left\|\frac{|G-\tilde{G}|}{v_{2}}\right\|_{L^{s^{\prime}}\left(\Omega, v_{2}\right)}\right)\left\|u_{1}-u_{2}\right\|_{X} .
\end{aligned}
$$

Hence, with $\gamma=\min \left\{\alpha_{p}, \tilde{\alpha}_{p}\right\}$, we obtain

$$
\begin{aligned}
& \gamma\left\|u_{1}-u_{2}\right\|_{X}^{p} \leq \alpha_{p} \int_{\Omega}\left|\Delta\left(u_{1}-u_{2}\right)\right|^{p} \omega_{1} d x+\tilde{\alpha}_{p} \int_{\Omega}\left|\nabla\left(u_{1}-u_{2}\right)\right|^{p} \omega_{2} d x \\
& \leq\left(C_{\Omega}\left\|\frac{f-\tilde{f}}{\omega_{2}}\right\|_{L^{p^{\prime}}\left(\Omega, \omega_{2}\right)}+M_{2}\left\|\frac{|G-\tilde{G}|}{v_{2}}\right\|_{L^{s^{\prime}}\left(\Omega, v_{2}\right)}\right)\left\|u_{1}-u_{2}\right\|_{X} .
\end{aligned}
$$

Therefore,

$$
\left\|u_{1}-u_{2}\right\|_{X} \leq \frac{1}{\gamma^{1 /(p-1)}}\left(C_{\Omega}\left\|\frac{f-\tilde{f}}{\omega_{2}}\right\|_{L^{p^{\prime}}\left(\Omega, \omega_{2}\right)}+M_{2}\left\|\frac{|G-\tilde{G}|}{v_{2}}\right\|_{L^{s^{\prime}}\left(\Omega, v_{2}\right)}\right)^{1 /(p-1)}
$$


Corollary 9. Assume $2 \leq q, s<p<\infty$. Let the assumptions of Theorem 7 be fulfilled, and let $\left\{f_{m}\right\}$ and $\left\{G_{m}\right\}$ be sequences of functions satisfying $\frac{f_{m}}{\omega_{2}} \rightarrow \frac{f}{\omega_{2}}$ in $L^{p^{\prime}}\left(\Omega, \omega_{2}\right)$ and $\left\|\frac{\left|G_{m}-G\right|}{v_{2}}\right\|_{L^{s^{\prime}}\left(\Omega, v_{2}\right)} \rightarrow 0$ as $m \rightarrow \infty$. If $u_{m} \in X$ is a solution of the problem

$$
\left(P_{m}\right)\left\{\begin{array}{l}
L u_{m}(x)=f_{m}(x)-\operatorname{div}\left(G_{m}(x)\right), \text { in } \Omega, \\
u_{m}(x)=\Delta u_{m}(x)=0, \text { in } \partial \Omega,
\end{array}\right.
$$

then $u_{m} \rightarrow u$ in $X$ and $u$ is a solution of problem $(P)$.

Proof. By Corollary 8 we have

$$
\left\|u_{m}-u_{r}\right\|_{X} \leq \frac{1}{\gamma^{1 /(p-1)}}\left(C_{\Omega}\left\|\frac{f_{m}-f_{r}}{\omega_{2}}\right\|_{L^{p^{\prime}\left(\Omega, \omega_{2}\right)}}+M_{2}\left\|\frac{\left|G_{m}-G_{r}\right|}{v_{2}}\right\|_{L^{s^{\prime}}\left(\Omega, v_{2}\right)}\right)^{1 /(p-1)} .
$$

Therefore $\left\{u_{m}\right\}$ is a Cauchy sequence in $X$. Hence, there is $u \in X$ such that $u_{m} \rightarrow u$ in $X$. We have that $u$ is a solution of problem $(P)$. In fact, since $u_{m}$ is a solution of $\left(P_{m}\right)$, for all $\varphi \in X$ we have

$$
\begin{aligned}
& \int_{\Omega}|\Delta u|^{p-2} \Delta u \Delta \varphi \omega_{1} d x+\int_{\Omega}|\Delta u|^{q-2} \Delta u \Delta \varphi v_{1} d x+\int_{\Omega}|\nabla u|^{p-2}\langle\nabla u, \nabla \varphi\rangle \omega_{2} d x+\int_{\Omega}|\nabla u|^{s-2}\langle\nabla u, \nabla \varphi\rangle v_{2} d x \\
& =\int_{\Omega}\left(|\Delta u|^{p-2} \Delta u-\left|\Delta_{m}\right|^{p-2} \Delta u_{m}\right) \Delta \varphi \omega_{1} d x+\int_{\Omega}\left(|\Delta u|^{q-2} \Delta u-\left|\Delta u_{m}\right|^{q-2} \Delta u_{m}\right) \Delta \varphi v_{1} d x \\
& +\int_{\Omega}\left(|\nabla u|^{p-2}\langle\nabla u, \nabla \varphi\rangle-\left|\nabla u_{m}\right|^{p-2}\left\langle\nabla u_{m}, \nabla \varphi\right\rangle\right) \omega_{2} d x \\
& +\int_{\Omega}\left(|\nabla u|^{s-2}\langle\nabla u, \nabla \varphi\rangle-\left|\nabla u_{m}\right|^{s-2}\left\langle\nabla u_{m}, \nabla \varphi\right\rangle\right) v_{2} d x \\
& +\int_{\Omega}\left|\Delta u_{m}\right|^{p-2} \Delta u_{m} \Delta \varphi \omega_{1} d x+\int_{\Omega}\left|\Delta u_{m}\right|^{q-2} \Delta u_{m} \Delta \varphi v_{1} d x+\int_{\Omega}\left|\nabla u_{m}\right|^{p-2}\left\langle\nabla u_{m}, \nabla \varphi\right\rangle \omega_{2} d x \\
& +\int_{\Omega}\left|\nabla u_{m}\right|^{s-2}\left\langle\nabla u_{m}, \nabla \varphi\right\rangle v_{2} d x \\
= & I_{1}+I_{2}+I_{3}+I_{4}+\int_{\Omega} f_{m} \varphi d x+\int_{\Omega}\left\langle G_{m}, \nabla \varphi\right\rangle d x \\
= & I_{1}+I_{2}+I_{3}+I_{4}+\int_{\Omega} f \varphi d x+\int_{\Omega}\langle G, \nabla \varphi\rangle d x+\int_{\Omega}\left(f_{m}-f\right) \varphi d x+\int_{\Omega}\left\langle G_{m}-G, \nabla \varphi\right\rangle d x
\end{aligned}
$$

where

$$
\begin{aligned}
& I_{1}=\int_{\Omega}\left(|\Delta u|^{p-2} \Delta u-\left|\Delta u_{m}\right|^{p-2} \Delta u_{m}\right) \Delta \varphi \omega_{1} d x, \\
& I_{2}=\int_{\Omega}\left(|\Delta u|^{q-2} \Delta u-\left|\Delta u_{m}\right|^{q-2} \Delta u_{m}\right) \Delta \varphi v_{1} d x, \\
& I_{3}=\int_{\Omega}\left(|\nabla u|^{p-2}\langle\nabla u, \nabla \varphi\rangle-\left|\nabla u_{m}\right|^{p-2}\left\langle\nabla u_{m}, \nabla \varphi\right\rangle\right) \omega_{2} d x, \\
& I_{4}=\int_{\Omega}\left(|\nabla u|^{s-2}\langle\nabla u, \nabla \varphi\rangle-\left|\nabla u_{m}\right|^{s-2}\left\langle\nabla u_{m}, \nabla \varphi\right\rangle\right) v_{2} d x .
\end{aligned}
$$

We have that:

(1) By Lemma 5 (a) there exists $C_{p}>0$ such that

$$
\begin{aligned}
\left|I_{1}\right| & \leq\left.\int_{\Omega}|| \Delta u\right|^{p-2} \Delta u-\left|\Delta u_{m}\right|^{p-2} \Delta u_{m}|| \Delta \varphi \mid \omega_{1} d x \\
& \leq C_{p} \int_{\Omega}\left|\Delta u-\Delta u_{m}\right|\left(|\Delta u|+\left|\Delta u_{m}\right|\right)^{p-2}|\Delta \varphi| \omega_{1} d x .
\end{aligned}
$$

Let $r=p /(p-2)$. Since $\frac{1}{p}+\frac{1}{p}+\frac{1}{r}=1$, by the Generalized Hölder inequality we obtain

$$
\left|I_{1}\right| \leq C_{p}\left(\int_{\Omega}\left|\Delta u-\Delta u_{m}\right|^{p} \omega_{1} d x\right)^{1 / p}\left(\int_{\Omega}|\Delta \varphi|^{p} \omega_{1} d x\right)^{1 / p}\left(\int_{\Omega}\left(|\Delta u|+\left|\Delta u_{m}\right|\right)^{(p-2) r} \omega_{1} d x\right)^{1 / r}
$$




$$
\leq C_{p}\left\|u-u_{m}\right\|_{X}\|\varphi\|_{X}\left\||\Delta u|+\left|\Delta u_{m}\right|\right\|_{L^{p}\left(\Omega, \omega_{1}\right)}^{(p-2)} .
$$

Now, since $u_{m} \rightarrow u$ in $X$, then exists a constant $M>0$ such that $\left\|u_{m}\right\|_{X} \leq M$. Hence,

$$
\left\||\Delta u|+\left|\Delta u_{m}\right|\right\|_{L^{p}\left(\Omega, \omega_{1}\right)} \leq\|u\|_{X}+\left\|u_{m}\right\|_{X} \leq 2 M .
$$

Therefore,

$$
\left|I_{1}\right| \leq C_{p}(2 M)^{p-2}\left\|u-u_{m}\right\|_{X}\|\varphi\|_{X}=C_{1}\left\|u-u_{m}\right\|_{X}\|\varphi\|_{X} .
$$

Analogously, there exists a constant $C_{3}$ such that

$$
\left|I_{3}\right| \leq C_{3}\left\|u-u_{m}\right\|_{X}\|\varphi\|_{X}
$$

(2) By Lemma 5 (a) there exists a positive constant $C_{q}$ such that

$$
\begin{aligned}
\left|I_{2}\right| & \leq\left.\int_{\Omega}|| \Delta u\right|^{q-2} \Delta u-\left|\Delta u_{m}\right|^{q-2} \Delta u_{m}|| \Delta \varphi \mid v_{1} d x \\
& \leq C_{q} \int_{\Omega}\left|\Delta u-\Delta u_{m}\right|\left(|\Delta u|+\left|\Delta u_{m}\right|\right)^{q-2}|\Delta \varphi| v_{1} d x .
\end{aligned}
$$

Let $\alpha=q /(q-2)$ (if $2<q<p<\infty)$. Since $\frac{1}{q}+\frac{1}{q}+\frac{1}{\alpha}=1$, by the Generalized Hölder inequality we obtain

$$
\begin{aligned}
\left|I_{2}\right| & \leq C_{q}\left(\int_{\Omega}\left|\Delta u-\Delta u_{m}\right|^{q} v_{1} d x\right)^{1 / q}\left(\int_{\Omega}|\Delta \varphi|^{q} v_{1} d x\right)^{1 / q}\left(\int_{\Omega}\left(|\Delta u|+\left|\Delta u_{m}\right|\right)^{(q-2) \alpha} v_{1} d x\right)^{1 / \alpha} \\
& =C_{q}\left\|\Delta u-\Delta u_{m}\right\|_{L^{q}\left(\Omega, v_{1}\right)}\|\Delta \varphi\|_{L^{q}\left(\Omega, v_{1}\right)}\left\||\Delta u|+\left|\Delta u_{m}\right|\right\|_{L^{q}\left(\Omega, v_{1}\right)}^{q-2} .
\end{aligned}
$$

Now, by Remark 3(a) and (9) we have

$$
\begin{aligned}
\left|I_{2}\right| & \leq C_{q} M_{1}\left\|\Delta u-\Delta u_{m}\right\|_{L^{p}\left(\Omega, \omega_{1}\right)} M_{1}\|\Delta \varphi\|_{L^{p}\left(\Omega, \omega_{1}\right)} M_{1}^{q-2}\left\||\Delta u|+\left|\Delta u_{m}\right|\right\|_{L^{p}\left(\Omega, \omega_{1}\right)}^{q-2} \\
& \leq C_{q} M_{1}^{q}\left\|u-u_{m}\right\|_{X}\|\varphi\|_{X}(2 M)^{q-2} \\
& =C_{2}\left\|u-u_{m}\right\|_{X}\|\varphi\|_{X} .
\end{aligned}
$$

Analogously, if $2<s<p<\infty$, there exists a positive constant $C_{4}$ such that

$$
\left|I_{4}\right| \leq C_{4}\left\|u-u_{m}\right\|_{X}\|\varphi\|_{X} .
$$

In case $q=2$ and $s=2$, we have $\left|I_{2}\right|,\left|I_{4}\right| \leq M_{1}^{2}\left\|u-u_{m}\right\|_{X}\|\varphi\|_{X}$.

Therefore, we have $I_{1}, I_{2}, I_{3}, I_{4} \rightarrow 0$ when $m \rightarrow \infty$.

(3) We also have

$$
\left|\int_{\Omega}\left(f_{m}-f\right) \varphi d x+\int_{\Omega}\left\langle G_{m}-G, \nabla \varphi\right\rangle d x\right|\left(C_{\Omega}\left\|\frac{f_{m}-f}{\omega_{2}}\right\|_{L^{p^{\prime}}\left(\Omega, \omega_{2}\right)}+M_{2}\left\|\frac{\left|G_{m}-G\right|}{v_{2}}\right\|_{L^{s^{\prime}}\left(\Omega, v_{2}\right)}\right)\|\varphi\|_{X} \rightarrow 0,
$$

when $m \rightarrow \infty$.

Therefore, in (8), we obtain when $m \rightarrow \infty$ that

$$
\begin{aligned}
& \int_{\Omega}|\Delta u|^{p-2} \Delta u \Delta \varphi \omega_{1} d x+\int_{\Omega}|\Delta u|^{q-2} \Delta u \Delta \varphi v_{1} d x+\int_{\Omega}|\nabla u|^{p-2}\langle\nabla u, \nabla \varphi\rangle \omega_{2} d x \\
& +\int_{\Omega}|\nabla u|^{s-2}\langle\nabla u, \nabla \varphi\rangle v_{2} d x=\int_{\Omega} f \varphi d x+\int_{\Omega}\langle G, \nabla \varphi\rangle d x,
\end{aligned}
$$

i.e., $u$ is a solution of problem $(P)$. 
Example 1. Let $\Omega=\left\{(x, y) \in \mathbb{R}^{2}: x^{2}+y^{2}<1\right\}, \omega_{1}(x, y)=\left(x^{2}+y^{2}\right)^{-1 / 2}, \omega_{2}(x, y)=\left(x^{2}+y^{2}\right)^{-1 / 4}\left(\omega_{i} \in A_{4}\right.$, $p=4$ and $q=s=3), v_{1}(x, y)=\left(x^{2}+y^{2}\right)^{-1 / 3}, v_{2}(x, y)=\left(x^{2}+y^{2}\right)^{1 / 8}, f(x, y)=\frac{\cos (x y)}{\left(x^{2}+y^{2}\right)^{1 / 6}}$ and $G(x, y)=$ $\left(\frac{\sin (x+y)}{\left(x^{2}+y^{2}\right)^{1 / 6}}, \frac{\sin (x y)}{\left(x^{2}+y^{2}\right)^{1 / 6}}\right)$. By Theorem 7 , the problem

$$
\left\{\begin{array}{l}
\Delta\left[\left(x^{2}+y^{2}\right)^{-1 / 2}|\Delta u|^{2} \Delta u+\left(x^{2}+y^{2}\right)^{-1 / 3}|\Delta u| \Delta u\right] \\
-\operatorname{div}\left[\left(x^{2}+y^{2}\right)^{-1 / 4}|\nabla u|^{2} \nabla u+\left(x^{2}+y^{2}\right)^{-1 / 8}|\nabla u| \nabla u\right] \\
=f(x)-\operatorname{div}(G(x)), \text { in } \Omega \\
u(x)=\Delta u=0, \text { in } \partial \Omega
\end{array}\right.
$$

has a unique solution $u \in W^{2,4}\left(\Omega, \omega_{1}\right) \cap W_{0}^{1,4}\left(\Omega, \omega_{2}\right)$.

Author Contributions: All authors contributed equally to the writing of this paper. All authors read and approved the final manuscript.

Conflicts of Interest: "The authors declare no conflict of interest."

\section{References}

[1] Cavalheiro, A. C. (2013). Existence and uniqueness of solutions for some degenerate nonlinear Dirichlet problems. Journal of Applied Analysis, 19(1), 41-54.

[2] Cavalheiro, A. C. (2017). Existence and uniqueness of solution for a class of nonlinear degenerate elliptic equation in weighted Sobolev spaces. Acta Universitatis Sapientiae, Mathematica, 9(1), 26-44.

[3] Cavalheiro, A. C. (2018). Topics on Degenerate Elliptic Equations. Lambert Academic Publishing, Germany.

[4] Fabes, E. B., Kenig, C. E., \& Serapioni, R. P. (1982). The local regularity of solutions of degenerate elliptic equations. Communications in Statistics-Theory and Methods, 7(1), 77-116.

[5] García-Cuerva, J., \& De Francia, J. R. (2011). Weighted norm inequalities and related topics (Vol. 116). Elsevier.

[6] Heinonen, J., Kipelainen, T., \& Martio, O. (2018). Nonlinear potential theory of degenerate elliptic equations. Courier Dover Publications.

[7] Muckenhoupt, B. (1972). Weighted norm inequalities for the Hardy maximal function. Transactions of the American Mathematical Society, 165, 207-226.

[8] Turesson, B. O. (2000). Nonlinear potential theory and weighted Sobolev spaces (Vol. 1736). Springer Science \& Business Media.

[9] Stein, E. M. (1993). Harmonic Analysis. Princenton University Press, New Jersey.

[10] Torchinsky, A. (1986). Real-variable methods in harmonic analysis (Vol. 123). Academic Press.

[11] Drábek, P., Kufner, A., \& Nicolosi, F. (2011). Quasilinear elliptic equations with degenerations and singularities (Vol. 5). Walter de Gruyter.

[12] Gilbarg, D., \& Trudinger, N. S. (2015). Elliptic partial differential equations of second order. springer.

[13] Chipot, M. (2009). Elliptic equations: an introductory course. Springer Science \& Business Media.

[14] Zeidler, E. (2013). Nonlinear Functional Analysis and Its Applications: II/B: Nonlinear Monotone Operators. Springer Science \& Business Media. 